\title{
The impact of social norm change strategies on smokers' quitting behaviours
}

\author{
Xueying Zhang, David W Cowling, Hao Tang
}

California Department of Public Health, Tobacco Control Program, Sacramento, California, USA

\section{Correspondence to}

Ms Xueying Zhang, California Department of Public Health

Tobacco Control Program, MS 7206, P.O. Box 997377

Sacramento, CA 95899-7377, USA

xueying.zhang@cdph.ca.gov

Received 27 January 2009 Accepted 11 February 2010

This paper is freely available online under the BMJ Journals unlocked scheme, see http:// tobaccocontrol.bmi.com/site/ about/unlocked.xhtml

\section{ABSTRACT}

Objective Using a social norm change paradigm model that reflects the California Tobacco Control Program's (CTCP) priorities, we compare the strength of the relationship of the social norm constructs to key smoking behavioural outcomes.

Methods Social norm constructs that correspond to CTCP's priority areas were created from selected California Adult Tobacco Survey knowledge, attitude and belief questions using confirmatory factor analysis. We then examined the relationship between these constructs and quitting behaviours using logistic regression.

Results The secondhand smoke (SHS) and countering pro-tobacco influences'(CPTI) constructs followed a dose-response curve with quitting behaviours. Respondents who rated high on the SHS construct were about $70 \%$ more likely to have made a recent quit attempt in the last 12 months and about $100 \%$ more likely to intend to quit in the next 6 months than respondents who rated low on the SHS construct. For $\mathrm{CPTI}$, respondents who rated high on this construct were $67 \%$ more likely to have made a recent quit attempt in the last 12 months and $62 \%$ more likely to have intentions to quit in the next 6 months than respondents who rated low on the CPTI construct.

Conclusion Social norm change constructs represent CTCP's priorities and are strongly related to desired individual behaviour outcomes. This analysis provides strong support for the framework underlying CTCP-namely, that changing social norms affects behaviour change at the individual level through changing population-level smoking-related behaviours.

\section{INTRODUCTION}

In 1988, a ballot initiative (Proposition 99) increased the tobacco excise tax by US $\$ 0.25$ and created the first state-mandated comprehensive tobacco control programme, the California Tobacco Control Program (CTCP). ${ }^{1}$ Ecological and econometric analyses have demonstrated an association between the implementation of CTCP and reductions in adult smoking prevalence and adult per capita cigarette consumption in California. ${ }^{2}$ Currently California has the second lowest adult smoking prevalence 4 and lowest per capita cigarette consumption in the USA. ${ }^{5}$ Longterm health outcomes, including reductions in tobacco-related cancer incidence and heart disease incidence have also been linked to the implementation of CTCP. ${ }^{7}$ Incidence of lung and bronchus cancer is currently declining four times faster in California than the rest of the US. ${ }^{8}$ The success of CTCP and its unprecedented use of the social norm strategy for tobacco control efforts resulted in the
Centers for Disease Control and Prevention (CDC) in 1999 identifying CTCP as the example of best practices for comprehensive tobacco control and prevention programmes worldwide. ${ }^{9}$

CTCP was the first tobacco control programme to integrate a 'social norm change' approach as its core strategy to achieve its goals. CTCP's social norm change approach employs the Social Ecological Model to create systems-level changes in a range of organisations such as institutions, schools, entertainment venues, workplaces and government agencies. ${ }^{10-12}$ A social norm is defined as shared expectations of culturally appropriate and desirable behaviour. ${ }^{13}$ The goal of CTCP's social norm change approach is "to indirectly influence current and potential future tobacco users by creating a social milieu and legal climate in which tobacco becomes less desirable, less acceptable and less accessible". ${ }^{14}$

A Californian adult's tobacco-related social norms are primarily influenced by CTCP in two ways. The first is a direct result of CTCP's statewide, multilingual and multicultural mass media campaign. The second is the passing and implementation of local and statewide tobacco control policies that change an individual's expectations and preferences. An example of policy impact is the percentage of bar owners or staff working in stand-alone bars who prefer to work in a smoke-free environment increasing from 17.3 percent just after the implementation of a smoke-free bar law in 1998 to $50.9 \%$ in 2002. ${ }^{15}$

Under this social norm change paradigm, the CTCP, beginning in 1989, has focused its tobacco control activities on three priority areas: (1) countering pro-tobacco influences in the community (CPTI); (2) reducing exposure to secondhand smoke (SHS); and (3) reducing the availability of tobacco (Tobacco Availability). The fourth priority area is 'supporting cessation efforts', which has been used as a necessary complement to the social norm change paradigm. ${ }^{16}$ Specifically, CPTI activities curb tobacco product retail advertisements and marketing practices, tobacco industry sponsorship and depiction of tobacco products in the entertainment industry. SHS activities employ a policy and advocacy approach to restrict smoking in public and private places; and Tobacco Availability activities enforce existing laws that prohibit selling tobacco to minors, eliminate free sampling and license tobacco retailers.

Our analyses are conducted on the adult population as CTCP is unique in tobacco control by primarily focusing its efforts on adults. Although there is a school component that concentrates on curricula and tobacco-free schools that is administered by the California Department of Education, 
CTCP is based on the theoretical frameworks that adults are the most important role models in a youth's life, Furthermore adults are policymakers that determine community-wide exposure to SHS, tobacco industry promotions and the enforcement of laws.

The relationship among tobacco control interventions, attitudes, beliefs and behaviour outcomes are described by the CDC's logic model. ${ }^{17}$ This logic model has been supported by findings from youth-oriented mass media interventions in Florida, Minnesota, and other national efforts. These studies have linked specific interventions with changes in smoking behaviour through a dose-response causal pathway of attitude change $^{1819}$ or brand equity. ${ }^{20}$

In this study, we examine whether the theoretical foundation of the CTCP can be described by social norm measures created from knowledge and attitude tobacco-related questions. Additionally, we examine which of the social norm constructs are important in predicting quitting behaviour outcomes.

\section{METHODS}

\section{Data source}

The California Adult Tobacco Survey (CATS) is an ongoing annual random digit dial telephone survey, conducted by CTCP since 1993, to monitor tobacco-related knowledge, attitudes, and behaviours. The survey monitors progress, provides formative evaluation data and is continuously updated to monitor areas of interest to CTCP. We combined data from 1997 to 2007 to obtain a sample of 50017 adult respondents, aged 18 and over. The Council of American Survey Research Organizations' average response rate was $45 \%$. More information can be found in the survey's technical documentation. ${ }^{21}$

\section{Measures}

\section{Quitting behaviour}

Current adult smokers were identified as those who had smoked at least 100 cigarettes in their lifetime and now smoke everyday or some days. Among current smokers, a quit attempt was defined as having stopped smoking for 1 day or longer because of trying to quit smoking during the past 12 months. A quit intention was defined as contemplating quitting smoking in the next 6 months.

\section{Knowledge/attitudes/beliefs towards tobacco issues}

In theory, attitudes and subjective social norms (the perception of peers' behaviour or attitudes), are distinct concepts predicting a specific behavioural intention, and subsequently predicting behavioural change. ${ }^{22}$ However, with the CTCP's social norm change paradigm and its ecological framework, the focus of the programme's interventions are not exclusively on smoking behaviours. For example, passing tobacco control policies to eliminate the hazardous effect of SHS is one of the priority areas of the CTCP, although this policy intervention is not necessarily focused on changing smoking behaviours directly. However, enacting such policies can lead to stronger anti-smoking norms because a natural implication of the policy is that smoking-related behaviours are not desirable. Therefore, attitude change towards this type of policy at the population level, especially over multiple years, can reasonably capture and represent change in smokingrelated social norms. This is based on the premise that social norms are a collective perception of appropriateness. ${ }^{13}$

We measure social norm change by including all 17 common tobacco-related knowledge, attitude and belief questions over multiple waves of CATS in our analysis. These questions cover a broad range of topics from SHS health effects and restrictions, to how respondents feel about restricting tobacco industry advertising and its promotions (dichotomous responses: yes, no; table 1). We chose to evaluate the social norm change paradigm using the three CTCP priority areas: SHS, CPTI and Tobacco Availability. Fourteen questions were used to form these three constructs. Additionally, other questions about tobacco product regulation which are of interest but not a focus of the programme were used to create a fourth construct.

\section{Demographic variables}

Race/ethnicity, age, gender, socio-economic status (SES) and a linear time variable were used to adjust the effect of latent variables in multivariate analysis. We created four categories for race/ethnicity: white, black, Hispanic and other. Age was grouped into 10-year segments. SES was defined as the following: (1) Low SES included individuals whose annual income fell below $\$ 25000$ and who had high school education or less; (2) High SES referred to individuals whose annual income was at least $\$ 50000$ and had obtained a college education or greater; (3) Middle SES included all other individuals. All variables were transformed to allow for nonlinearity by using log-odds of the outcomes.

\section{Confirmatory factor analysis}

Confirmatory factor analysis (CFA) was used to investigate whether the established dimensionality of attitudes and beliefs and its factor-loading pattern fit the data. Figure 1 presents the

Table 1 Latent factors and attitudinal questions

\begin{tabular}{|c|c|}
\hline Latent factors & $\begin{array}{l}\text { Tobacco related knowledge, } \\
\text { attitudinal or belief questions }\end{array}$ \\
\hline \multirow[t]{4}{*}{ SHS } & $\begin{array}{l}\text { Inhaling smoke from someone else's cigarette causes lung } \\
\text { cancer in a nonsmoker. }\end{array}$ \\
\hline & $\begin{array}{l}\text { Inhaling smoke from someone else's cigarette harms the } \\
\text { health of babies and children. }\end{array}$ \\
\hline & I prefer to eat in restaurants that are smoke free. \\
\hline & $\begin{array}{l}\text { All indoor worksites, including restaurants and cafeterias, } \\
\text { should be smoke free. }\end{array}$ \\
\hline
\end{tabular}

Availability

Minors caught buying cigarettes should be fined. Store owners should need a license to sell cigarettes (just like alcoholic beverages).

Cigarette vending machines should be totally prohibited. Local communities should strongly enforce laws that prevent people from selling cigarettes to minors.

CPTI

Advertising tobacco products at sports and athletic events
should be banned.
The ban on cigarette advertising should be extended to all
print and electronic media.
The tobacco industry should not be permitted to offer
products such as clothing or camping equipment in
exchange for coupons on cigarette packs.
The distribution of free tobacco samples or coupons to
obtain free samples by mail should not be permitted.
The tobacco industry spokespersons mislead the public
when they say tobacco is not addictive.
Tobacco advertising encourages young people to start
smoking.
The tobacco industry should be forced to put stronger
warnings on all their potentially harmful products.
Tobacco products should be treated like other foods and
drugs, with each package having full disclosure of
potentially harmful contents.
Tobacco products should be regulated as a drug by
a government agency such as the Food and Drug
Administration.

CPTI, countering pro-tobacco influences in the community; SHS, secondhand smoke. 
latent factors and the subsets of attitudinal variables of each factor. We used SHS, CPTI, tobacco availability and tobacco product regulation as the four underlying dimensions. These four latent factors were chosen using the CTCP's social norm change framework. To test the stability of the factor structure over years, and whether it is appropriate to combine multiple years' CATS data for future analysis, 11 survey years (1997-2007) were sorted into three time periods: 1997-2000, 2000-2003 and 2004-2007. CFA was conducted for each combined sample for each time period, and the factor loadings from the three models were compared. No meaningful difference was observed among the factor loadings of the three time periods. Because the factorloading pattern of attitudes and beliefs did not change over time, we combined data from 1997 to 2007 and fitted the CFA using the combined data set. The CFA analysis was conducted in SAS V.9.1 using the CALIS procedure with a set of structural equations described in the LINEOS statement.

The CFA yielded good fit indices: the Root Mean Square Error of Approximation Test, a measure of model residuals, was equal to 0.06 , and the Goodness Fit Index adjusted for degrees of freedom was 0.92 (0.90 and higher indicating good fit). ${ }^{23}$

We obtained the factor loading of each attitudinal variable on its corresponding latent factor from CFA (figure 1). Factor loading is the correlation coefficient between variables and latent factors and represents the percent of variance of each attitudinal variable explained by the latent factor. Although it is common to use a factor loading cutpoint of 0.3 or $0.4,{ }^{24}$ several attitudinal variables with smaller factor loadings were kept in the model. We reasoned that CFA tested whether the structure of latent variables fit the data; consequently, the significance of individual factor loading was not critical to the model. Additionally, our goal was not to create a parsimonious model but one that could be used to explain progress in the CTCP's priority areas.

In order to categorise the factor scores into groups, we scaled the factor scores to values between zero and 100 to allow for divergent social norms in the low and high groups. Based on the distribution of the four constructs, we categorised individuals into the low group if factor scores fell below 70 and high if above 90. For each factor, a high score meant respondents had attitudes and beliefs that were more closely aligned with desired tobacco control attitudes and beliefs.

Multivariate logistic regression was used to relate the latent variables with quitting behaviours of interest-a quit attempt in the last 12 months and intentions to quit in the next 6 months. The regression model also included other known predictors of quitting behaviours, including age, gender, race/ethnicity and SES.

\section{RESULTS}

As shown in figure 2, among the four social norm constructs, SHS norms consistently produced the highest scores and CPTI had the lowest scores over the 10-year period. The four constructs showed small incremental increases over time. Quitting behaviours also showed a similar upward trend during this period.

SHS attitudes were strongly related to smoking status $(A O R=0.19$ for high vs low; 95\% CI: 0.14, 0.24). In this instance, attitudes may not be a precursor of smoking behaviour as a large portion of these attitudes may be determined by an individual's smoking status. A stronger set of attitudes and beliefs for CPTI $(\mathrm{AOR}=0.67$ for high to low; 95\% CI: $0.53,0.86)$ were also strongly related to smoking status.

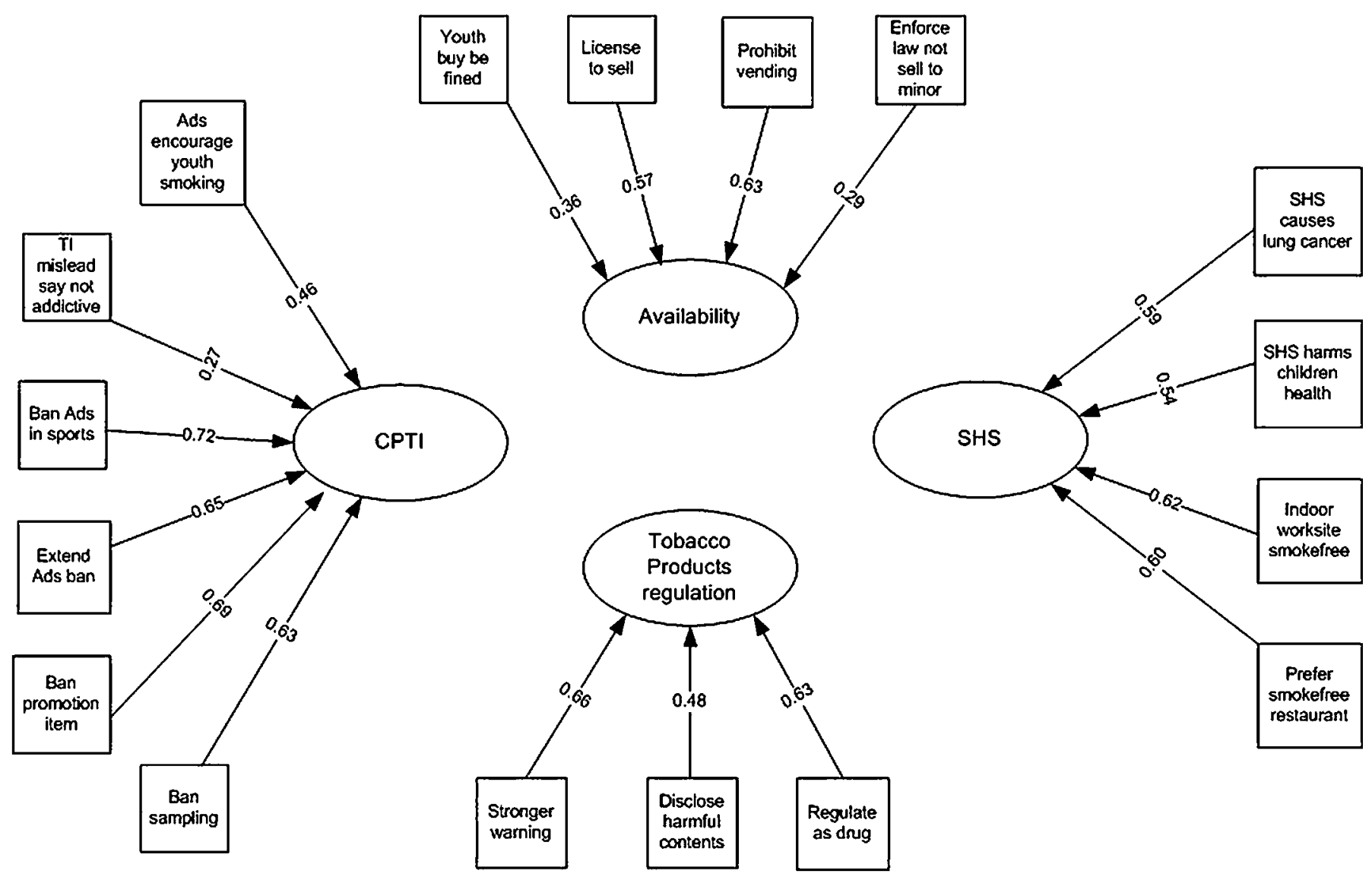

Figure 1 Latent structure and factor loadings for attitudes/beliefs from the California Adult Tobacco Survey 1997-2007. CPTI, countering protobacco influences in the community; SHS, secondhand smoke; TI, tobacco industry. 
SHS and CPTI norms demonstrated a dose-response relationship with having made a quit attempt and having intentions to quit. As shown in table 2, respondents with higher SHS scores were over 1.7 times more likely to have made a quit attempt in the last 12 months compared to respondents with low factor scores $(\mathrm{AOR}=1.7$ for high vs low; 95\% CI: 1.40, 2.07). Respondents with higher SHS scores were twice as likely to have an intention to quit in the next 6 months than respondents with low factor scores (AOR=2.0 for high vs low; 95\% CI: 1.55, 2.46).

Respondents with higher CPTI factor scores were about $70 \%$ more likely to have made a quit attempt in last 12 months than respondents with low factor scores $(\mathrm{AOR}=1.67$ for high vs low; $95 \%$ CI: $1.26,2.22)$ and 1.6 times more likely to have an intention to quit in next 6 months than respondents with low factor scores ( $A O R=1.62$ for high vs low; 95\% CI: 1.14, 2.30).

Tobacco product regulation was not associated with quit attempts $(\mathrm{AOR}=1.2$ for high vs low; $95 \% \mathrm{CI}$ : $0.85,1.60)$ or intentions to quit in the next 6 months $(A O R=1.1$ for high vs low; 95\% CI: $0.73,1.60)$. Also, Tobacco Availability was not associated with quit attempts (AOR=0.9 for high vs low; $95 \%$ CI: $0.66,1.26)$ or intentions to quit in the next 6 months $(\mathrm{AOR}=1.2$ for high vs low; 95\% CI: $0.83,1.86)$.

\section{DISCUSSION}

Tobacco-related knowledge, attitude and belief questions from a population-based survey were used as representative factors (latent variables) that are presumed to measure CTCP's progress in changing tobacco-related social norms and population-level smoking behaviours. CPTI and SHS were shown to be associated with quitting behaviours. Smokers with more positive attitudes towards CPTI and SHS reported more quit attempts and intentions. Programmatically, CTCP has had tremendous success in these two priority areas: reducing SHS exposure through implementing SHS laws locally and exposing the predatory marketing practices of the tobacco industry through a comprehensive, multicultural and multi-lingual advertising campaign. Although CTCP does not actively focus on individual behaviour change through promoting its cessation services, the programme's social norm change strategies still make smokers quit smoking.

Besides quitting behaviours, we examined the association between the constructs and smoking status. We found a strong

Table 2 Adjusted odds ratios for the association between attitude scales and quitting behaviors

\begin{tabular}{|c|c|c|c|c|}
\hline \multirow[b]{2}{*}{ Attitudes/beliefs scale level } & \multicolumn{2}{|c|}{ Quit attempt } & \multicolumn{2}{|c|}{ Quit intention } \\
\hline & $\overline{A O R} \dagger$ & $95 \% \mathrm{CL}$ & $\overline{\mathrm{AOR} \dagger}$ & $95 \% \mathrm{CL}$ \\
\hline \multicolumn{5}{|l|}{ SHS } \\
\hline High vs low & $1.70^{*}$ & $1.40,2.07$ & $1.95^{*}$ & $1.55,2.46$ \\
\hline Medium vs low & $1.20^{*}$ & $1.01,1.43$ & $1.54^{*}$ & $1.27,1.87$ \\
\hline \multicolumn{5}{|l|}{ Availability } \\
\hline High vs low & 0.91 & $0.66,1.26$ & 1.24 & $0.83,1.86$ \\
\hline Medium vs low & 1.08 & $0.88,1.32$ & 1.02 & $0.81,1.28$ \\
\hline \multicolumn{5}{|l|}{ CPTI } \\
\hline High vs low & $1.67^{*}$ & $1.26,2.22$ & $1.62^{*}$ & $1.14,2.30$ \\
\hline Medium vs low & $1.29^{*}$ & $1.05,1.59$ & $1.30^{*}$ & $1.02,1.65$ \\
\hline \multicolumn{5}{|l|}{ Tobacco product } \\
\hline High vs low & 1.17 & $0.85,1.60$ & 1.08 & $0.73,1.60$ \\
\hline Medium vs low & 1.19 & $0.97,1.45$ & 1.09 & $0.87,1.38$ \\
\hline
\end{tabular}

${ }^{*} \mathrm{p}<0.05$.

†Adjusted by age, gender, race, social economic status, year. CPTI, countering pro-tobacco influences in the community; $\mathrm{CL}$, confidence limit; SHS, secondhand smoke.

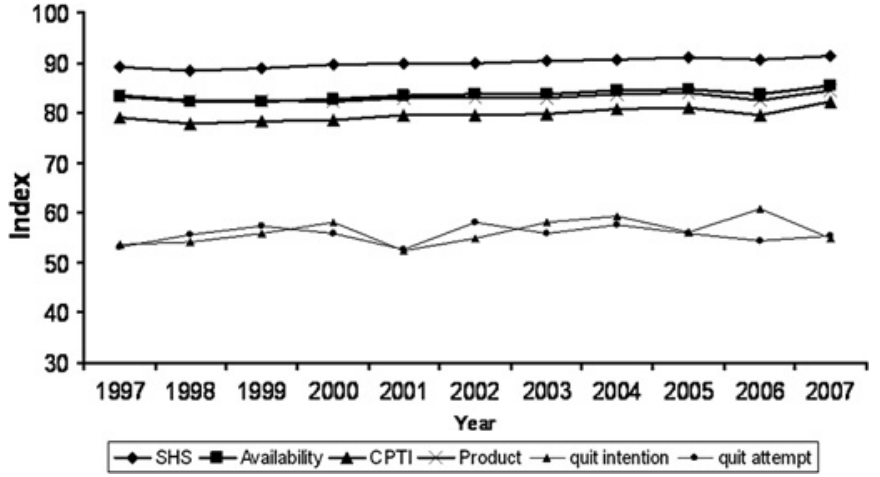

Figure 2 Social norm index trend from 1997 to 2007. CPTI, countering pro-tobacco influences in the community; SHS, secondhand smoke.

association between SHS constructs and smoking status. Because we used repeated cross-sectional surveys, it is not clear whether desired attitudes and beliefs determine smoking status or whether smoking status determines attitudes and beliefs. However, by focusing only on smokers we were able to control for smoking status as a major predictor of tobacco-related attitudes.

The CTCP has had and is currently achieving local programme success around Tobacco Availability; however, this area was not associated with quit attempts nor quit intentions. Attitudes about Tobacco Availability were not related to norms but were related to the enforcement of laws. Consequently, future attitude questions will need to tactfully address the CTCP's messages and activities around Tobacco Availability.

In California, the SHS construct in the future could be improved by including measures of items to address the expansion of outdoor SHS laws, such as on beaches and parks. Additionally, the introduction of harm reduction products necessitates adjustment to tobacco product regulation questions and potentially to the CPTI measures. Since we used data from 1997 to 2007, we could only examine the trend of the four constructs after 1997. But social norms prior to the first survey (1997) are also likely to have changed because the CTCP has been implementing the social norm change strategy since 1989.

We found a compelling link between the social norm change paradigm and quitting behaviours. This relationship provides evidence for theoretical underpinnings of this paradigm, demonstrates that the social norm change approach can lead to meaningful changes in tobacco-related health behaviours, and provides support for being an effective public health model applicable to other tobacco control programmes worldwide.

\section{What this paper adds}

The California Tobacco Control Program (CTCP) was the first tobacco control program to integrate a 'social norm change' approach as its core strategy to achieve its goals. This paper used a social norm change paradigm model to reflect CTCP's priorities and compared the strength of the relationship of the social norm constructs to key smoking behavioral outcomes. This paper provides strong support for the framework underlying CTCP - namely, that changing social norms affects behavior change at the individual level through changing population-level smoking-related behaviors. 
Acknowledgements We thank Dr Michael K Cummings for his invaluable comments.

Competing interests None.

Provenance and peer review Not commissioned; externally peer reviewed.

\section{REFERENCES}

1. Bal DG, Lloyd JC, Roeseler A, et al. California as a model. J Clin Oncol 2001;19 (Suppl 18):69S-73S.

2. Pierce JP, Gilpin EA, Emery SL, et al. Has the California tobacco control program reduced smoking? JAMA 1998;280:893-9.

3. Hu TW, Sung HY, Keeler TE. Reducing cigarette consumption in California: tobacco taxes vs. an anti-smoking media campaign. Am J Public Health 1995;85:1218-22.

4. Substance Abuse and Mental Health Services Administration. Results from the 2004 National Survey on Drug Use and Health: National Findings (Office of Applied Studies, NSDUH Series H-28, DHHS Publication No. SMA 05-4062). Rockville, MD: U.S. Department of Health and Human Services, 2005.

5. Orzechowski W, Walker RC. The tax burden on tobacco: historical compilation. Vol 40. Arlington, VA: Orzechowski \& Walker, 2005.

6. Centers for Disease Control and Prevention. Declines in lung cancer rates-California, 1988-1997. MWWR Morb Mortal Wkly Rep 2000;49:1066-9.

7. Fichtenberg CM, Glantz SA. Association of the California Tobacco Control Program with declines in cigarette consumption and mortality from heart disease. $N$ Engl J Med 2000;343:1772-7.

8. California Department of Public Health, Tobacco Control Program (2008). California Tobacco Control Update 2008.

9. Centers for Disease Control and Prevention. Best Practices for Comprehensive Tobacco Control Programs-August 1999. Atlanta, GA: U.S. Department of Health and Human Services, Centers for Disease Control and Prevention, National Center for Chronic Disease Prevention and Health Promotion, Office on Smoking and Health, 1999
10. McLeroy KR, Bibean D, Steckler A, et al. An ecological perspective on health programs. Health Educ 0 1988;15:351-77.

11. Stokols D. Translating social ecological theory into guidelines for community health promotion. Am J Health Promot 1996;10:282-98.

12. Brownson RC, Koffman DM, Novotny TE, et al. Environmental and policy interventions to control tobacco use and prevent cardiovascular disease. Health Educ 0 1995;22:478-98.

13. Marshall G. A dictionary of sociology. Oxford, UK: Oxford Express, 1998. http:// www.encyclopedia.com/doc/1088-norm.html (accessed 3 Feb 2010).

14. California Department of Health Services, Tobacco Control Section (1998) A model for change: the California experience in tobacco control.

15. Tang H, Cowling DW, Stevens CM, et al. Changes of attitudes and behaviors of bar owners and staff after a smoke-free bar law. Tob Control 2004;13:87-9.

16. California Department of Health Services, Tobacco Control Section (1998) California Tobacco Control Update 1998.

17. Starr G, Rogers T, Schooley M, et al. Key outcome indicators for evaluating comprehensive Tobacco Control Programs. Atlanta, GA: Centers for Disease Contro and Prevention, 2005

18. Sly DF, Trapido E, Ray S. Evidence of the dose effects of an antitobacco counteradvertising campaign. Prev Med 2002:35:511-18.

19. Arheart KL, Sly DF, Trapido EJ, et al. Assessing the reliability and validity of antitobacco attitudes/beliefs in the context of a campaign strategy. Prev Med 2004:39:909-18.

20. Evans WD, Price S, Blahut S. Evaluating the truth ${ }^{\circledR}$ brand. J Health Commun 2005:10:181-92

21. California Adult Tobacco Survey 2007 Documentation and Technical Report. http:// www.surveyresearchgroup.org/sub.php?page=projects_adult_tobacco laccessed Oct 2008).

22. Fishbein M, Ajzen I. Belief, attitude, intention, and behavior: an introduction to theory and research. Reading, MA: Addison-Wesley, 1975

23. Hu L, Bentler PM. Cutoff criteria for fit indexes in covariance structure analysis: conventional criteria versus new alternatives. SEM 1999;6:1-55.

24. SAS documentation. http://support.sas.com/91doc/getDoc/statug.hlp/factor sect16 htm (accessed 21 Jul 2006). 